\title{
DNA methylation and histone modifications regulate SOX11 expression in lymphoid and solid cancer cells
}

Lena Nordström, Elin Andersson², Venera Kuci', Elin Gustavsson', Karolina Holm³, Markus Ringnér ${ }^{3}$, Per Guldberg ${ }^{2}$ and Sara Ek ${ }^{1^{*}}$

\begin{abstract}
Background: The neural transcription factor SOX11 is present at specific stages during embryo development with a very restricted expression in adult tissue, indicating precise regulation of transcription. SOX11 is strongly up-regulated in some malignancies and have a functional role in tumorgenesis. With the aim to explore differences in epigenetic regulation of SOX11 expression in normal versus neoplastic cells, we investigated methylation and histone modifications related to the SOX11 promoter and the possibility to induce re-expression using histone deacetylase (HDAC) or EZH2 inhibitors.

Methods: The epigenetic regulation of SOX11 was investigated in distinct non-malignant cell populations $(n=7)$ and neoplastic cell-lines ( $n=42$ ) of different cellular origins. DNA methylation was assessed using bisulfite sequencing, methylation-specific melting curve analysis, MethyLight and pyrosequencing. The presence of H3K27me3 was assessed using ChIP-qPCR. The HDAC inhibitors Vorinostat and trichostatin A were used to induce SOX11 in cell lines with no endogenous expression.

Results: The SOX11 promoter shows a low degree of methylation and strong enrichment of H3K27me3 in non-malignant differentiated cells, independent of cellular origin. Cancers of the B-cell lineage are strongly marked by de novo methylation at the SOX11 promoter in SOX11 non-expressing cells, while solid cancer entities display a more varying degree of SOX11 promoter methylation. The silencing mark H3K27me3 was generally present at the SOX11 promoter in non-expressing cells, and an increased enrichment was observed in cancer cells with a low degree of SOX11 methylation compared to cells with dense methylation. Finally, we demonstrate that the HDAC inhibitors (vorinostat and trichostatin A) induce SOX11 expression in cancer cells with low levels of SOX11 methylation.

Conclusions: We show that SOX11 is strongly marked by repressive histone marks in non-malignant cells. In contrast, SOX11 regulation in neoplastic tissues is more complex involving both DNA methylation and histone modifications. The possibility to re-express SOX11 in non-methylated tissue is of clinical relevance, and was successfully achieved in cell lines with low levels of SOX11 methylation. In breast cancer patients, methylation of the SOX11 promoter was shown to correlate with estrogen receptor status, suggesting that SOX11 may be functionally re-expressed during treatment with HDAC inhibitors in specific patient subgroups.
\end{abstract}

Keywords: SOX11, DNA methylation, H3K27, Epigenetic regulation

\footnotetext{
*Correspondence: sara.ek@immun.lth.se

'Department of Immunotechnology, CREATE Health, Lund University, Lund, Sweden

Full list of author information is available at the end of the article
} 


\section{Background}

During embryonic development, cell-fate decisions and lineage commitment are regulated by both transcription factors and epigenetic mechanisms. The SOX protein family of transcription factors is known to act as important regulators of embryonic development, cellular fate determination and differentiation [1,2]. SOX11, a member of the SOXC subgroup, plays an important role in both embryonic and adult neurogenesis, and is proposed to regulate self-renewal of neuronal progenitor cells [3]. The expression of SOX11 is absent in most adult differentiated tissues, further supporting the role as a stem cell specific regulator [4]. SOX11 has been shown to be regulated by epigenetic events in pluripotent embryonic stem cells and is marked with both activating (H3K4me3) and repressive (H3K27me3) histone marks [5]. These bivalent marks are thought to keep developmentally important genes silenced, but poised for activation during lineage commitment [6]. Bivalent histone marks are often modified during cell differentiation so that only the active or repressive marks remain [7]. In agreement with this, peripheral B-cells that lack SOX11 have been reported to be strongly marked by H3K27me3 [8]. Interestingly, it has been shown that genes marked with Н3К27me3 are targets for de novo methylation in cancer [9]. This is supported by gene expression analysis of de novo methylated genes that show lack of expression already in unmethylated non-malignant tissues [10].

Aberrant regulation of SOX11 has been observed in several tumors, leading to expression of the protein or silencing through promoter DNA methylation. Upregulation of SOX11 has been reported in malignant glioma [11], medulloblastoma [12], mantle cell lymphoma (MCL) [13], as well as subsets of Burkitt's lymphoma [14], ovarian cancer [15] and breast cancer [16]. Aberrant promoter methylation of $S O X 11$ has been reported in most mature B-cell lymphomas except MCL, which express SOX11 [13] and where SOX11 has functional [17] and prognostic [18] roles. Moreover, the presence of SOX11 promoter methylation has been shown to be significantly higher in patients with lymph node metastasis compared to patients without metastasis in nasopharyngeal carcinoma [19]. SOX11 methylation was also used in a five-gene biomarker panel to detect bladder cancer at an early stage [20]. Thus, both SOX11 expression and methylation pattern correlate to clinical behaviour, which is of major interest in relation to the novel use of epigenetic drugs, enabling demethylation and/or reexpression of silenced genes.

In the present study, we aimed to further investigate the epigenetic regulation of SOX11 in non-malignant $(n=7)$ and neoplastic cell populations $(n=42)$ to possibly identify new clinical subgroups with an aberrant regulation and/or expression of SOX11. We show that non-malignant cells have a low degree of DNA methylation but that SOX11 is enriched with $\mathrm{H} 3 \mathrm{~K} 27 \mathrm{me} 3$. In neoplastic cells, the epigenetic regulation of SOX11 is more complex. Most B-cell lymphomas are heavily methylated in the $S O X 11$ promoter region while solid tumor cells show a more diverse methylation pattern. Furthermore, in breast cancer, we demonstrate a correlation between SOX11 methylation and clinical subtype.

As the use of histone deacetylase (HDAC) inhibitors in the clinic is continuously growing, we evaluated the effect of epigenetic drugs on SOX11 expression. We show that SOX11 expression could be induced in cells with low levels of methylation by HDAC but not EZH2 inhibitors.

\section{Methods}

\section{FACS sorting of non-malignant B-cell populations}

Pediatric tonsils $(n=6)$ (Lund University Hospital, Lund, Sweden) were used as the source of normal nonmalignant B-cells and collected under written informed consent by parents or guardians. The use was ethically approved by the regional Lund/Malmo committee (Dnr 242/2006). The lymphocyte population was isolated by Ficoll gradient centrifugation. Viable B-cell populations were sorted based on $\mathrm{CD}$ markers as follows: naïve B-cells (CD3-, CD19+, IgD+, CD38-), GC B-cells (CD3-, CD19+, IgD-, CD38+) and memory B-cells (CD3, CD19+, IgD-, CD27+). FACS analysis of sorted populations confirmed a purity of $>95 \%$.

\section{Cell culture}

Forty two cell lines with different tumor origins were used to study the epigenetic regulation of SOX11. These included mantle cell lymphoma $(n=10)$, follicular lymphoma $(n=3)$, diffuse large B-cell lymphoma $(n=2)$, Burkitt's lymphoma $(n=4)$, epithelial ovarian cancer $(n=5)$, breast cancer $(n=8)$, lung cancer $(n=3)$, glioma cancer $(n=5)$ and neuroblastoma cell lines $(n=2)$. Two glioma cell lines were established from patient tissues and approved by the Local Ethical Board of the University of Lund, Sweden, serial no. LU307-98. Informed consent was obtained. To protect patient anonymity, tumor samples were coded to GBM-LU60 and GBM-LU93. All cell lines were cultured at $37 \mathrm{C}^{\circ}$ in a humidified atmosphere of $5 \%$ $\mathrm{CO}_{2}$. Details about cell culture media and supplier are shown in Additional file 1.

\section{DNA preparation and bisulfite conversion}

DNA was extracted and purified using QIAamp DNA Mini Kit (Qiagen, Hilden, Germany) followed by quantification on NanoDrop (NanoDrop technologies, Wilmington, DE, USA). All samples were bisulfite treated with EZ DNA Methylation Kit (Zymo Research, Irvine, CA, USA) according to manufacturer's protocol. Five hundred nanograms of 
DNA were used for each bisulfite conversion and converted samples were eluted in $20 \mu \mathrm{l}$ buffer.

\section{DNA methylation analysis of FACS sorted populations of non-malignant B-cells}

The CpG island adjacent to the SOX11 transcription start site was PCR amplified with primers specific for bisulfite treated DNA and subcloned into the TOPO-TA cloning vector as previously described [17]. Sequencing of individual alleles was made at GATC Biotech (Konstanz, Germany). The sequencing files were analyzed using $\mathrm{BiQ}$ Analyzer software [21] http://biq-analyzer.bioinf.mpi-inf. mpg.de/index.php. Sequences with poor conversion rates $(<95 \%)$ and identical clones, possibly generated in the PCR reaction, were removed. Data presentation images and methylation statistics were generated using the BDPC web server [22].

\section{DNA methylation microarrays of human breast cells}

DNA from human mammary fibroblasts, epithelial cells, and endothelial cells, as well as mesenchymal bone marrow stem cells (ScienCell Research Laboratories, CA, USA) was analyzed. Bisulfite conversion of $500 \mathrm{ng}$ genomic DNA was performed using the EZ DNA Methylation kit (Zymo Research, Orange, CA, USA) following the manufacturer's protocol. We hybridized $200 \mathrm{ng}$ in $4 \mu \mathrm{l}$ to the Infinium HumanMethylation450K BeadChip array (Illumina, San Diego, CA). The array includes five CpG sites within the SOX11 promoter (cg07065111, cp08432727, cg08526991, cg12312988, cg13667638, see Additional file 2). Bisulfite conversion and hybridization to arrays were performed by the SCIBLU facility, Lund, Sweden. Raw intensities for methylated $(\mathrm{M})$ and unmethylated $(\mathrm{U})$ signal were extracted from Illumina's GenomeStudio. Beta-values were calculated as $\mathrm{M} /(\mathrm{M}+\mathrm{U})$. Beta-values with detection $\mathrm{p}$ value $>0.05$ or with less than 3 beads for a signal were set as missing values. For each sample we performed a peakbased correction of Illumina I and II chemical assays similar to et al. [23]. For both assays we smoothed the beta values (Epanechnikov smoothing kernel) to estimate unmethylated and methylated peaks, respectively; and the unmethylated peak was moved to 0 and the methylated peak to 1 using linear scaling, with beta-values in between stretched accordingly. Beta-values below 0 were set back to 0 and values above 1 were set to 1 .

\section{Analysis of the ENCODE project data}

ChIP-seq data (H3K27me3 and H3K4me3) from human mammary epithelial cells were downloaded from the ENCODE project [24]. The sequence files were visualized with the Integrative Genomics Viewer (IGV).

\section{Methylation-specific melting curve analysis (MS-MCA) of tumor cell-lines}

Primers used in MS-MCA amplify all types of epialleles that later are discriminated during the melting stage of the analysis, enabling a qualitative description of the sample. Primers for MS-MCA [25] were designed to amplify a sequence 273 bp upstream of SOX11 transcription start site, containing $28 \mathrm{CpG}$ sites (See Additional file 2). Primers used were: 5'-TTTTAATTTTTTGTAGAAGGAG-3' and 5'-CCTTCCAAACTACACACAA-3'. Amplification and melting analysis was carried out on LightCycler 2.0 (Roche, Basel, Switzerland) using Fast Start DNA Master SYBR Green kit (Roche). Profiles of melting curves for fully methylated and unmethylated sequence was established using in vitro methylated DNA (IVM, Millipore, Billerica, MA, USA) and whole genome amplified DNA (WGA), derived with GenomiPhi V2 DNA amplification kit (GE Healthcare, Little Chalfont, Buckinghamshire, United Kingdom), respectively. Examples of how MS-MCA results was interpreted are shown in Additional file 3.

\section{MethyLight Analysis of tumor cell-lines}

MethyLight is a highly sensitive quantitative method amplifying highly methylated alleles. Data is normalized to a reference sample and presented as percent methylated reference (PMR). MethyLight analysis [26] of the SOX11 promoter region was performed on Roche LightCycler 480 realtime PCR using Lightcycler 480 Probes Master Kit (Roche) with primers 5'-GGTAGGAGTTACGAGTCGG AGAGA-3 and 5'ACTACGATCGCGACAAAAAAAAC3 ' and probe 5'-[6FAM]TCGGGTTGTTTCGATCG [MGBNFQ]-3' [20]. The assay was validated with bisulfite-treated DNA from cell lines unmethylated for SOX11 and non-bisulfite treated genomic DNA (human genome DNA, Roche). A dilution series of fully methylated control (in vitro methylated DNA, IVM, Millipore) were included in each reaction. A separate reaction for repetitive sequence ALUC4 [27] was performed on each sample to control for input DNA. All reactions were done in duplicate and an average value of the concentration was used to determine DNA methylation level in each sample. Percent methylated reference, PMR were calculated according to the formula: $\mathrm{PMR}=(([\mathrm{SOX} 11$ sample $] /[\mathrm{ALUC}$ 4sample])/([SOX11IVM]/[ALUC4IVM])) x 100.

\section{Pyrosequencing}

The 28 CpG sites investigated with MS-MCA were sequenced in bisulfite treated samples using the PyroMark Q24 platform (Qiagen, Hilden, Germany). One set of amplification primers (fwd primer: 5'ATGATATTT TGATAATTAGTTGAG-3' and rev primer: 5'-[Btn] CCTTCCAAACTACACACAA-3') and two sequencing primers (seq primer 1: 5'-AGAGAGATTTTAATTTTTTG TAGA-3', seq primer 2: 5'-AGTAGGAGAGAGGGGTT-3' ) 
were used to cover all 28 sites. PCR was carried out in a final volume of $25 \mu \mathrm{l}$ containing PCR buffer (Qiagen), 200 $\mu \mathrm{M}$ each of dNTP, $0.4 \mu \mathrm{M}$ each primer and $1 \mathrm{U}$ of Taq HotStarTaq DNA polymerase (Qiagen). Sequencing was performed using PyroMark Gold Q24 reagents (Qiagen). Analysis of the results was carried out with the PyroMark Q24 software (Qiagen). Results from at least two sequencing events were used to calculate the methylation level at each CpG site. In vitro methylated DNA (IVM, Millipore) and whole genome amplified DNA (WGA) derived with GenomiPhi V2 DNA amplification kit (GE Healthcare), were used as fully methylated and unmethylated control, respectively.

\section{RNA isolation and Real-Time QPCR assessment of SOX11}

SOX11 mRNA expression was investigated using realtime quantitative PCR. Cells were lysed and cDNA synthesized using iScriptTM Synthesis Kit (BIORAD, Hercules, CA, USA) according to manufacture instructions. Amplified cDNA was analyzed in triplicates using SsoFastTM EvaGreen ${ }^{\circ}$ Supermix with Low ROX (BIO-RAD) with primers specific for either SOX11: 5'GGTGGATAAGGATTTGGATTCG-3' and 5'-GCTCC GGCGTGCAGTAGT-3, or for the house-keeping gene GAPDH: 5'AGTAGAGGCAGGGATGATG-3' and 5'TGGTATCGTGGAAGGACTC-3'.

\section{Western blot analysis of SOX11 and EZH2}

$8 \times 10^{6}$ cells were harvested and protein extract preparation, quantification was performed as previously described by Gustavsson et al. [17]. Protein lysate $(20 \mu \mathrm{g})$ were run on a NuPAGE 10\% Bis-Tris gel (Invitrogen) and blotted on to a PVDF membrane using the iBLot ${ }^{\circ}$ Dry Blotting System (Invitrogen). The membrane was blocked in 5\% Milk/PBS before incubating with primary antibodies. Protein expression were assessed using the following antibodies: SOX11 monoclonal antibody [28], mouse anti GAPDH antibody (G8795, Sigma-Aldrich, St Louis, MO, USA) and EZH2 monoclonal antibody (Clone 11/EZH2, BD Transduction Laboratories, Franklin Lakes, NJ, USA). A HRP-labeled anti mouse antibody (P0260, DAKO, Glostrup, Denmark) was used for detection. Proteins were developed using SuperSignal West Femto Max Sensitivity Substrate (Pierce Biotechnology, Rockford, IL, USA) and images retrieved using a CCDcamera (Odyssey FC Imager from LI-COR Biosciences UK Ltd, Cambridge, England).

\section{Analysis of TCGA data}

Level 2 methylation data from breast tumor samples from the TCGA data portal https://tcga-data.nci.nih.gov/ tcga/ were processed as described for the human breast cells. Selecting unique female patients resulted in 669 tumors for further analysis. For 661 of the 669 samples, level 3 RNA sequencing data consisting of normalized gene counts was available. The transformation log2 (normalized gene count +1 ) was used to generate gene expression levels for further analysis. Pearson correlation between corrected beta values and gene expression levels were used to investigate association between promoter methylation and gene expression levels. ER status was available for 599 of the tumors; 139 were ER-negative and 460 were ER-positive. Two-sided Wilcoxon tests were used to test for differences between ER-positive and ER-negative tumors.

\section{Histone ChIP}

Chromatin immunoprecipitation of H3K27me3 and H3K4me3 bound regions were performed with the HighCell ChIP kit (Diagenode, Liege, Belgium) according to the protocol of the manufacturer. Antibodies against H3K27me3 (ab6002, Abcam, Cambridge, MA, USA) and rabbit IgG (Diagenode) were used in the ChIP experiments. Primers targeting the promoter of GAPDH (Diagenode) and SOX11 (fwd: 5'-GAGAGCTTGGAAGCGGAGA-3' rev: 5'-AGTCTGGGTCGCTCTCGTC-3') were used.

\section{Treatment with Trichostatin A, Vorinostat and GSK343}

Cells $\left(1 \times 10^{6}\right)$ were seeded into a 6 -well plate and cultured for 24 hours before drug treatment. Each cell line was treated with $0,0.5$ and $5 \mu \mathrm{M}$ trichostatin A (SigmaAldrich), 0, 0.5 and $5 \mu \mathrm{M}$ Vorinostat (Selleck, Houston, TX, USA) or 0, 10 and $20 \mu \mathrm{M}$ GSK343 (Sigma-Aldrich ). For all treatments, DMSO was used as a vehicle control. After $24 \mathrm{~h}$ (Trichostatin A and Vorinostat) or $72 \mathrm{~h}$ (GSK343) of incubation, cells were harvested, protein lysate prepared and western blot performed as described above.

\section{Results}

The aims of the present study were to explore the epigenetic regulation of SOX11 in non-malignant and neoplastic cells of various origins and to assess the possibility to reexpress SOX11 upon treatment with HDAC inhibitors.

\section{Epigenetic profiling of SOX11 in non-malignant cells}

The epigenetic regulation of SOX11 in non-malignant cells has until now been widely unexplored. To assess if the previously observed SOX11 promoter methylation and histone modifications in B cell lymphomas are a consequence of tumorgenesis or merely reflect the epigenetic status of the normal counterpart, non-malignant mature B cells from three differentiation stages, including naive, germinal center (GC) and memory B-cells, were FACS-sorted from tonsils $(\mathrm{n}=6)$.

The SOX11 promoter contains four CpG islands, where the island most proximal to the transcription start site has been shown to be determinative for SOX11 expression [17]. Consequently, $28 \mathrm{CpG}$ sites within this CpG 
island were sequenced after bisulfite conversion (Figure 1). The fraction of methylated CpGs was calculated over all sequenced alleles (10-20 per sample) and revealed a low degree of SOX11 methylation although a trend of increased methylation during differentiation was observed (Figure 1B). However, major inter-individual variations were observed, especially among the GC B cells. Using ChIPqPCR, we further observed that the SOX11 promoter showed a strong enrichment of H3K27me3 in all three Bcell populations (Figure $1 \mathrm{C}$ ). As non-malignant reference tissue for solid tumors, DNA from human mammary fibroblasts, epithelial cells, and endothelial cells, as well as mesenchymal stem cells were analyzed on Illumina 450K methylation arrays (as part of a larger study). Information on five CpGs within the SOX11 promoter was available. Analysis of the mammary cell types revealed that the two sites closest to the transcription start site were completely unmethylated while three sites upstream displayed a low degree of methylation (Figure 1D). Of note, cg12312988 is not located within a CpG island (see Additional file 2). Finally, ChIP-seq data (H3K27me3 and H3K4me3) on human mammary epithelial cells (downloaded from the ENCODE project [24]) showed strong enrichments of H3K27me3 over H3K4me3 on the SOX11 promoter (Figure 1E).

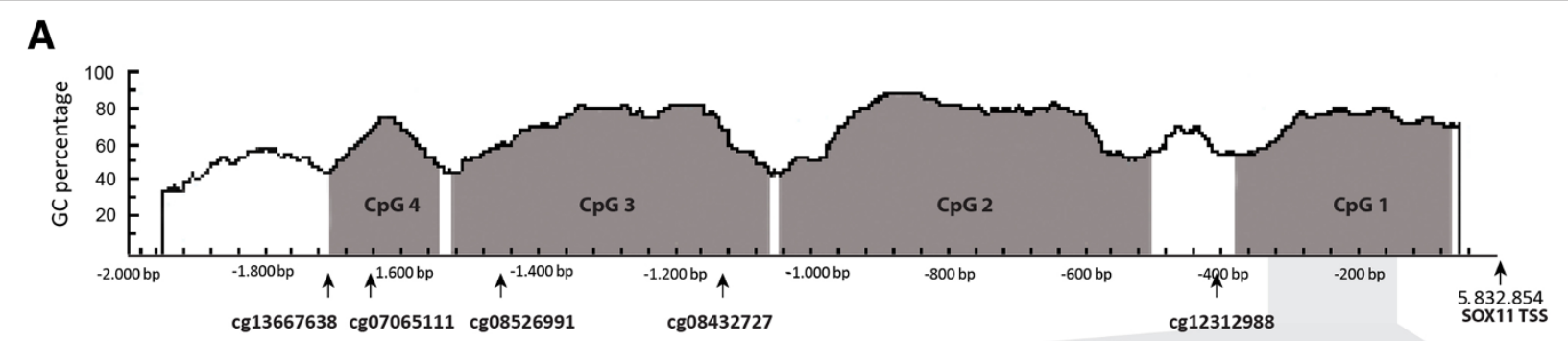

Illumina $450 \mathrm{~K}$ methylation array sites

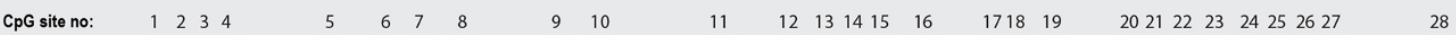

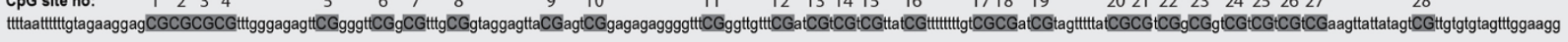

B

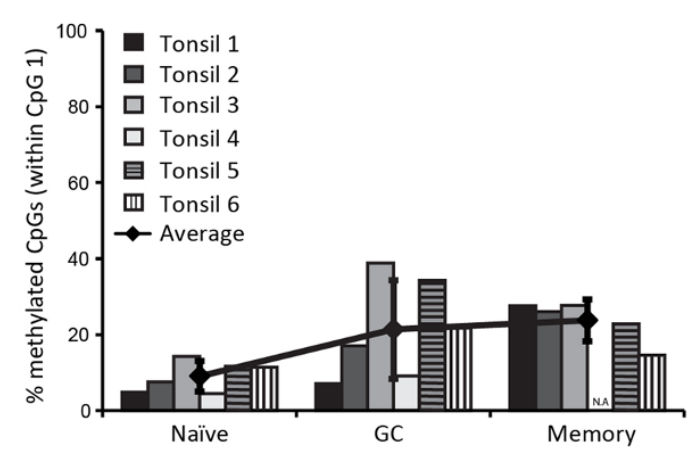

C

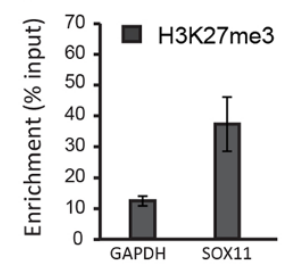

D

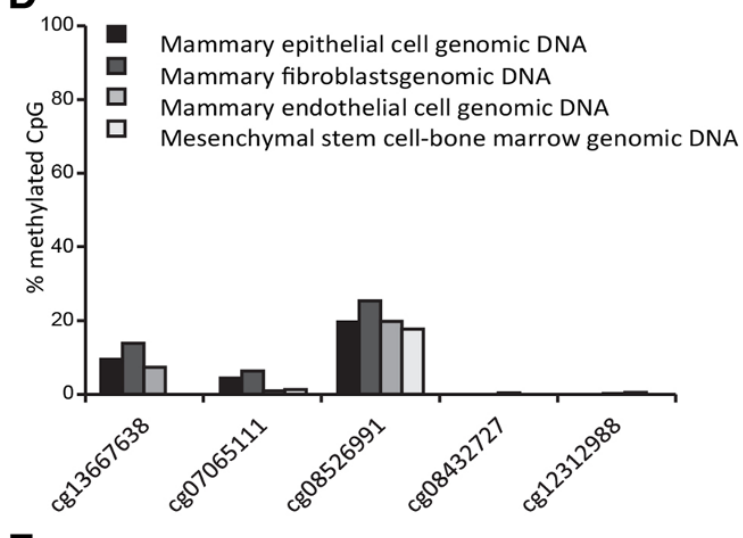

E

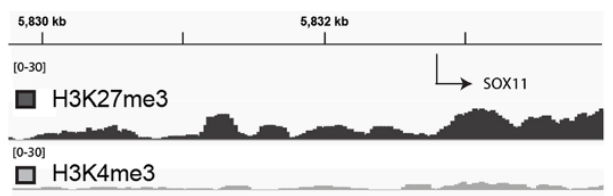

Figure 1 Epigenetic profiling of SOX11 in normal cells. (A) The SOX11 promoter 2000 bp upstream of transcription start site contains four CpG islands with analyzed CpG sites marked. (B) Mean SOX11 promoter methylation within 28 CpG sites close to the transcription start site. (C) Enrichment of repressive H3K27me3, determined by ChIP-qPCR, within the SOX11 promoter in naïve, GC and memory B-cells. (D) Methylation status of five CpG sites within the SOX11 promoter, measured with Illumina $450 \mathrm{~K}$ methylation array, for several types of non-malignant mammary cell-types. (E) Enrichment of active (H3K4me3) and repressive (H3K27me3) histone marks in primary human mammary epithelial cells. ChIP-seq data was extracted from the ENCODE project. 


\section{DNA methylation status of SOX11 in lymphoid and solid tumors}

To explore the difference between non-malignant reference tissue and neoplastic cells, we further investigated the methylation status of SOX11 in 42 cell lines (Table 1) representing a wide range of human tumors with subgroups known to express SOX11, including lymphoid malignancies $(n=19)$, ovarian cancer $(n=5)$, breast cancer $(n=8)$, lung cancer $(n=3)$, brain cancers $(n=5)$ and neuroblastoma $(n=2)$. To determine DNA methylation by complementary methods, MethyLight and methylationspecific melting curve analysis (MS-MCA) were used. The MethyLight and MS-MCA assays covered 8/28 and 28/28 CpG sites previously investigated in non-malignant mature B-cells, respectively (see Additional file 2). Overall, a good agreement between MethyLight and MS-MCA was observed in our sample set (Figure 2A and B), although calculated PMR values were significantly lower compared to absolute values derived from bisulfite sequencing of the same cell lines [17]. In agreement with public data $[8,17,29]$, we show that SOX11 is de novo methylated in all Burkitt's lymphomas, follicular lymphomas and diffuse large B-cell lymphomas. In mantle cell lymphomas that express SOX11, the promoter is generally unmethylated (Figure 2A and B). Solid tumors show a much more diverse methylation pattern within the SOX11 promoter (Figure 2C and D), possibly reflecting clinical subtypes with an altered epigenetic regulation.

\section{Correlation between promoter methylation and SOX11 expression}

To explore the correlation between SOX11 promoter methylation and expression, each cell line was analyzed by RT-qPCR and western blot. An inverse correlation was observed between $S O X 11$ promoter methylation and gene expression for both lymphoid and solid tumor cells (Spearman's correlation $\rho=-0.71 ; \mathrm{p}<0.001$ and $\rho=-0.75$;

Table 1 Cell-lines investigated for SOX11 expression and promoter methylation

\begin{tabular}{ll}
\hline Neoplasm & Cell-line \\
\hline $\begin{array}{l}\text { Mantle cell } \\
\text { lymphoma }\end{array}$ & REC-1, GRANTA-519, JEKO-1, SP53, MINO, Z138, \\
Follicular lymphoma & DOHH-2, RL, SC-1 \\
$\begin{array}{l}\text { Diffuse large B-cell } \\
\text { lymphoma }\end{array}$ & WSU-NHL, SU-DHL-8 \\
Burkitt's lymphoma & BJAB, RAJI, DAUDI, RAMOS \\
Breast cancer & JIMT-1, PMC-42, MDA-MB-231, SK-BR-3, T47D, \\
& BT474, BT9549, L56Br-C1 \\
Ovarian cancer & OVCAR-3, TOV-112D, ES-2, A2780, A2780-CP7 \\
Lung cancer & A549, DMS-114, NCI-H1299 \\
Glioma & HS683, LN-18, U87MG, GMB-LU93, GMB-LU60 \\
Neuroblastoma & SK-N-SH, KCN69n \\
\hline
\end{tabular}

$\mathrm{p}<0.001$, respectively) (Figure $3 \mathrm{~A}$ and $\mathrm{B}$ ). SOX11 protein was detected in $7 / 8$ (88\%) MCL cell-lines with an unmethylated promoter (Figure 3C), while only 6/14 (43\%) solid cancer cell lines with an unmethylated promoter had detectable levels of the protein (Figure 3D). As expected, none of the cell lines with a methylated promoter expressed SOX11 mRNA or protein with the exception of BJAB. Using MS-MCA, we show that BJAB has a monoallelic methylation of the SOX11 promoter, explaining the observed co-existence of a methylated promoter and expression of mRNA/protein (Figure 2A and Figure $3 \mathrm{~A}$ and $\mathrm{C}$ ). Pyrosequencing was further used to investigate if specific $\mathrm{CpG}$ sites are important for SOX11 silencing in cell lines with low-to-intermediate methylation (as determined by MS-MCA and MethyLight). Data show that even at very low level of overall methylation, CpGs close to transcription start site are significantly methylated compared to expressing cell lines with a completely unmethylated promoter (Additional file 4). Finally, we demonstrate a correlation between SOX11 methylation, expression and subtypes in primary breast cancers. Breast cancer methylation data and RNA-seq data were downloaded from The Cancer Genome Atlas (TCGA) and show that SOX11 methylation is more abundant in estrogen recptor (ER) positive tumors $(n=460)$ compared to ER negative tumors $(n=139)$ (Figure 4A) with a strong anti-correlation between methylation and expression in each CpG site (Figure 4B and Table 2).

\section{Chromatin immunoprecipitation of H3K27me3 in neoplastic cells}

As discussed above, normal cells have a strong enrichment of the silencing histone mark H3K27me3 on the promoter of SOX11 but show a low degree of promoter methylation (Figure 1). In contrast, many neoplastic cell lines show a high degree of SOX11 promoter methylation (Figure 3). To investigate if neoplastic cell lines with a low degree of methylation depend on H3K27me3 to silence SOX11, cell lines with a low or high degree of methylation were investigated to determine the enrichment of H3K27me3 at the SOX11 promoter. The biological variation was significant, exemplified by the major variation in enrichment of the positive control, TSH $2 B$. In two cell lines, DMS-114 and $\mathrm{KCN}-69$ n, the positive control showed such low levels of enrichment that data on SOX11 cannot be interpreted. $G A P D H$ was used as a negative control and background levels were set to the largest observed $G A P D H$ value. We show that H3K27me3 at the SOX11 promoter is enriched in several cell lines, including JIMT-1, LN-18 and JVM-2. However, SK-BR-3 and HS683 show a low enrichment compared to the positive control $(T S H 2 B)$ and are likely dependent on other epigenetic regulation than promoter methylation or H3K27me3 to silence SOX11 (Figure 5A). For comparison, three methylated cell-lines were analyzed 


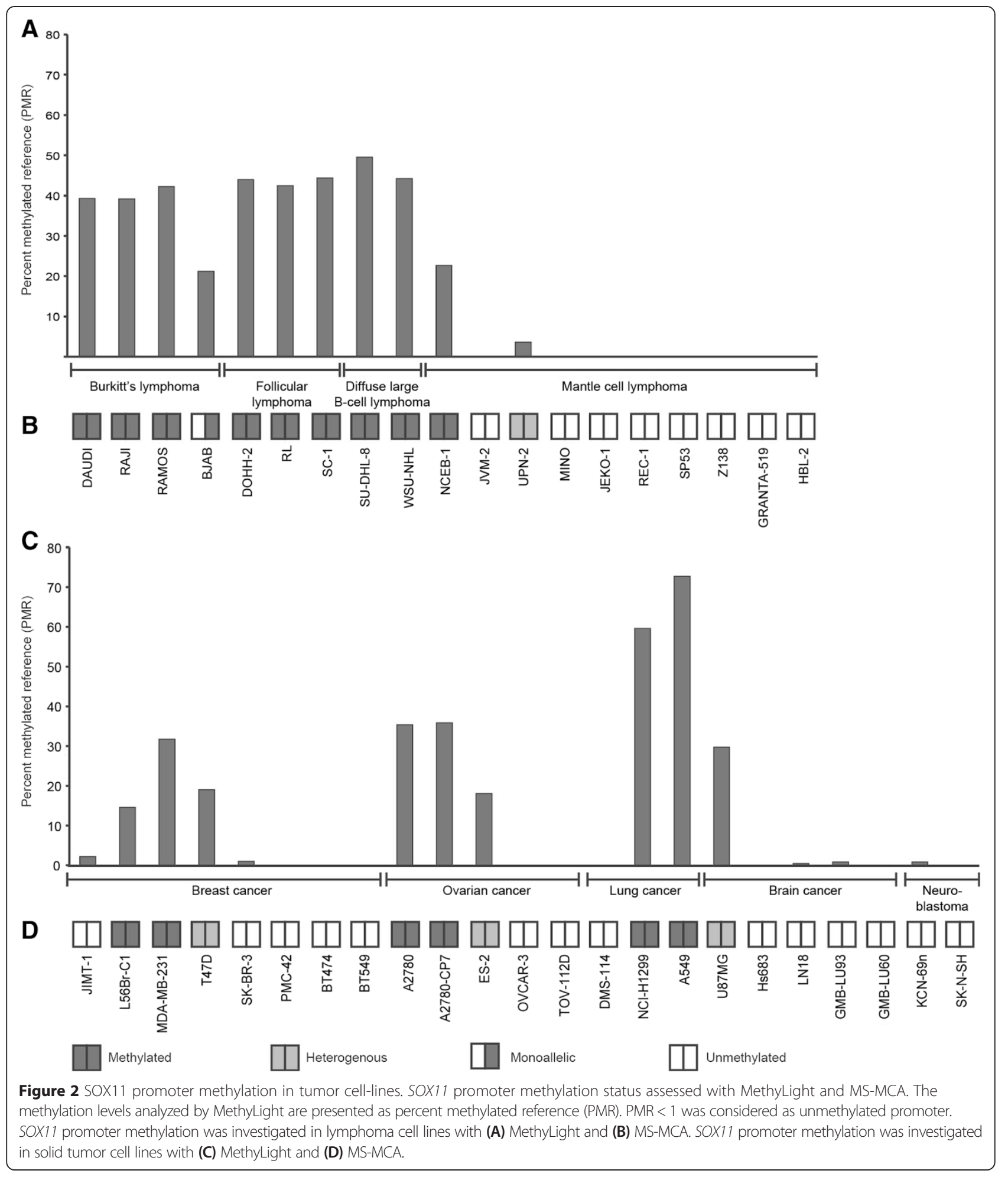

and all three cell lines, DOHH-2, RAJI and A2780-CP7 showed low enrichment of H3K27me3 at the SOX11 promoter compared to the positive control, indicating that methylation of the promoter may correlate to loss of repressive histone marks (Figure 5B).
Trichostatin A and Vorinostat induce expression of SOX11 in unmethylated cells

Since the expression of SOX11 was shown to be regulated by repressive histone marks with or without an additional layer of methylation, we investigated the potential of two 


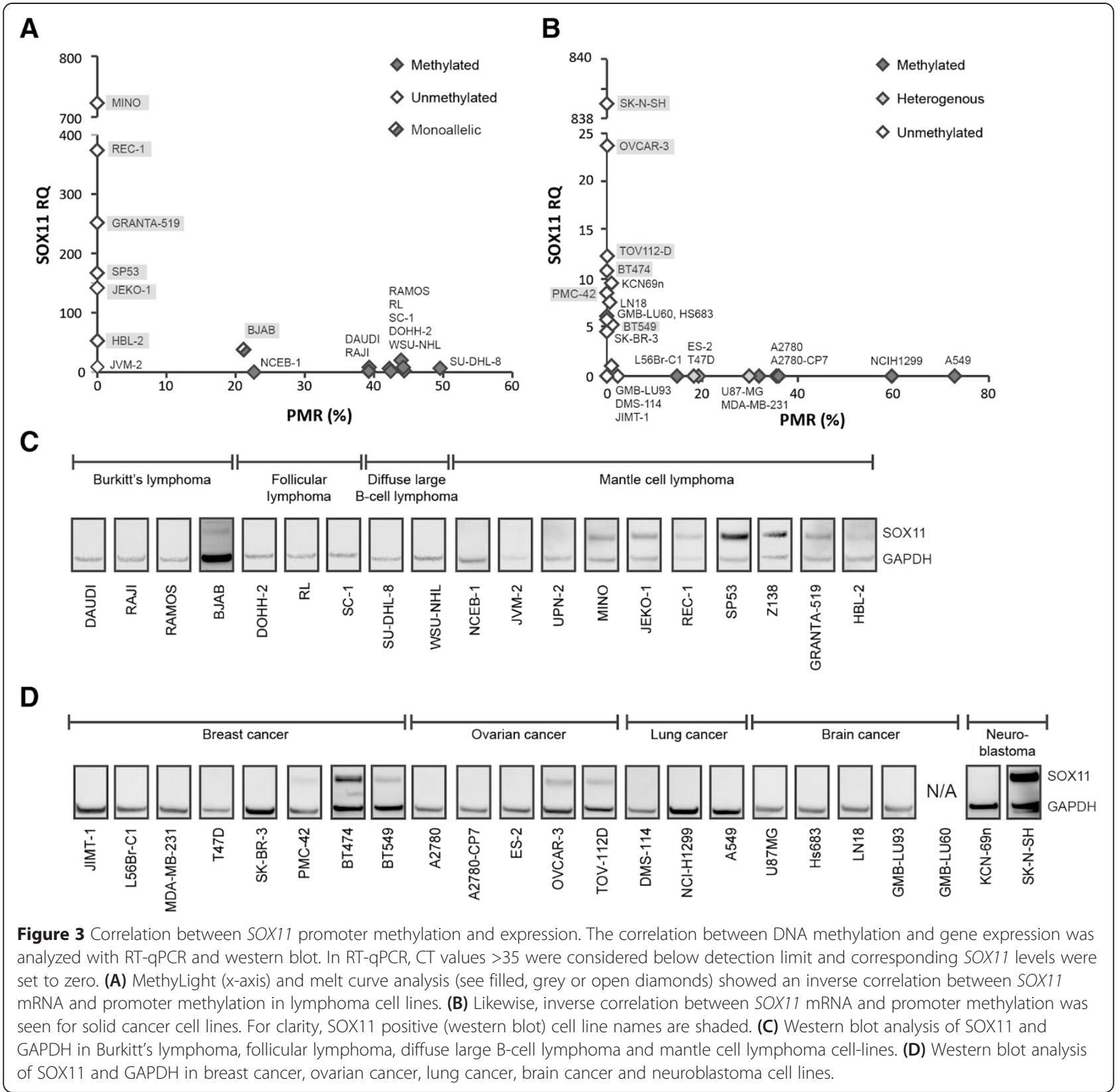

commonly used HDAC inhibitors vorinostat and trichostatin A (TSA), to re-express SOX11. The demethylating agent 5-aza-2'-deoxycytidine arrest proliferation already at low concentrations in lymphoid cells, and demethylation could thus not be assessed. Cell lines with no detectable levels of endogenous SOX11 with an unmethylated promoter were treated with $0,0.5$, and $5 \mu \mathrm{M}$ of TSA or vorinostat for 24 hours. Both TSA and vorinostat induced SOX11 expression in SK-BR-3, JIMT-1 and KCN-69n. TSA was more potent than vorinostat, and showed protein induction already at $0.5 \mu \mathrm{M}$ (Figure 6A). However, none of the drugs was able to induce SOX11 expression in DMS-114 and JVM-2, the latter in contrast to previous results [8]. Although some HDAC inhibitors have been reported to have a demethylating effect [30-32], we show that SOX11 expression could not be induced in any of the strongly methylated cell lines assessed, including RAJI, A2780-CP7 and DOHH-2 (Additional file 5A). Additionally, we demonstrate that $\mathrm{EZH} 2$, the enzyme responsible for H3K27 tri-methylation, was down-regulated upon TSA treatment (Figure 6B). To investigate if downregulation of EZH2 is enough to induce expression of SOX11, the cell-lines were further treated with GSK343, an EZH2 inhibitor. However, despite EZH2 downregulation in the majority of evaluated cell-lines, SOX11 was not re-expressed (Additional file 5B). 


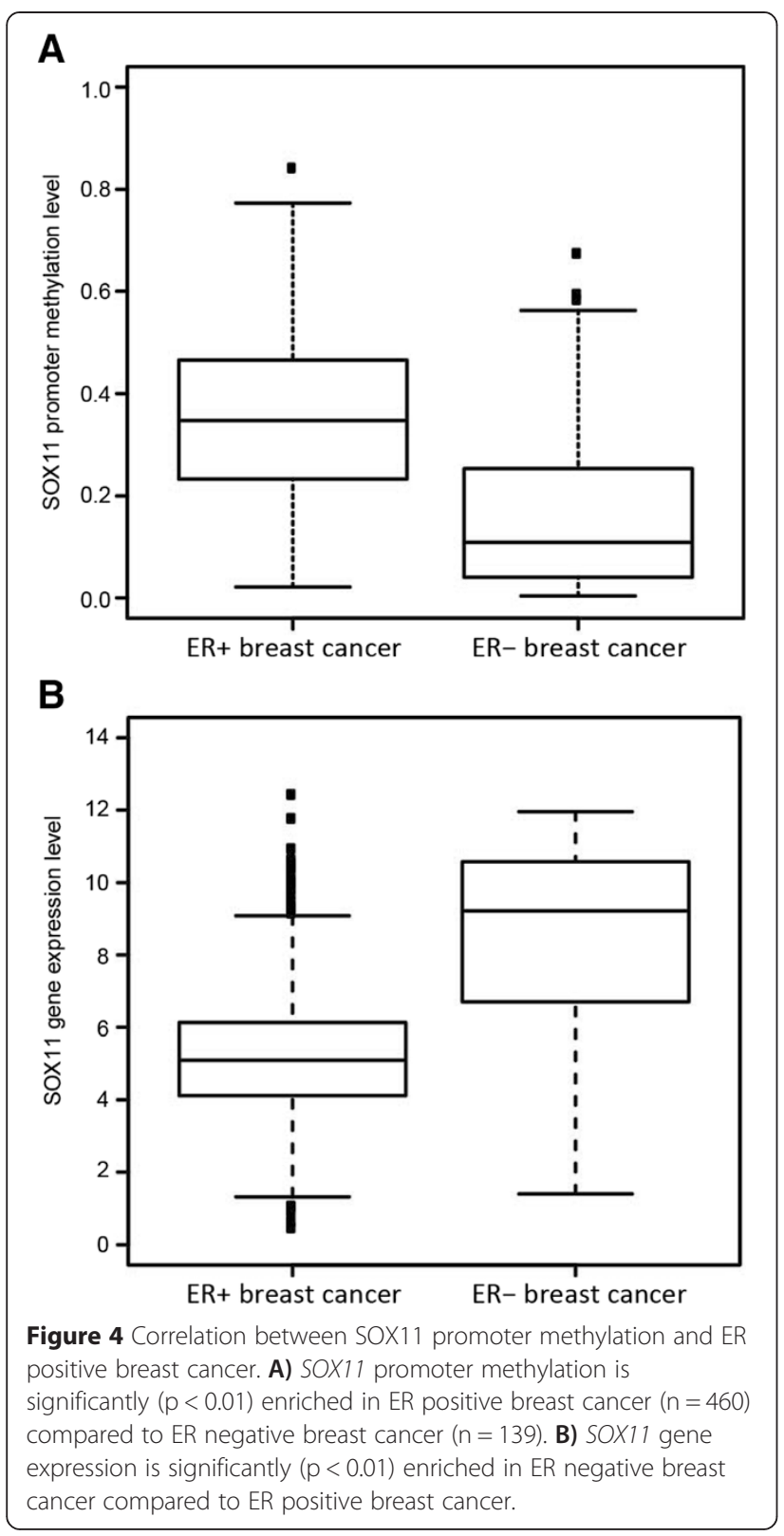

Table 2 Correlation between SOX11 promoter methylation and gene expression in primary breast cancers

\begin{tabular}{lll}
\hline CpG site & Correlation & Std methylation \\
\hline cg13667638 & -0.40 & 0.24 \\
cg07065111 & -0.49 & 0.25 \\
cg08526991 & -0.50 & 0.26 \\
cg08432727 & -0.39 & 0.20 \\
cg12312988 & -0.16 & 0.12 \\
\hline
\end{tabular}

Std gene expression $=2.42$.

\section{Discussion}

In non-malignant cells, epigenetic mechanisms are used to ensure flexible gene expression during development but later also permanent silencing of genes in differentiated tissues. Many human neoplasias display an altered epigenetic pattern, with overexpression or mutations of histone modifying enzymes and increased promoter methylation, leading to silencing of tumor suppressors [33]. These alterations are often reversible and the use of epigenetic drugs has become an attractive option to reprogram and sensitize cancer cells. During the last decade, both DNA demethylating agents (azacitidine and decitabine) and HDAC inhibitors (vorinostat and romidepsin) have been approved by FDA for use in myelodysplastic syndromes and cutaneous T-cell lymphoma, respectively [34-37]. Thus, epigenetic drugs have shown success in treatment of lymphoproliferative diseases, and several novel epigenetic drugs are currently in clinical trials for use in solid cancers.

With the growing interest in using epigenetic therapies in both hematological and solid malignancies, studies of novel epigenetically regulated genes are warranted and will provide (i) basic understanding, (ii) potential to use information on methylation in biomarker panels and (iii) opportunity to re-activate tumor suppressor functions or induce cancer stem cell differentiation [38-40] using novel epigenetic treatment strategies. We and others have during recent years shown that SOX11 is a diagnostic [13], prognostic [18,41,42], and functional biomarker in classical MCL [17], indolent MCL [43,44], ovarian cancer [15] and astrocytic gliomas [45]. SOX11 protein expression has been shown to correlate to increased and decreased survival in different tumor entities, emphasizing different function depending on molecular and cellular context.

Furthermore, initial epigenetic investigations shown that SOX11, which is a transcription factor normally expressed in a stage-specific manner during embryo development, has a bivalent histone mark (H3K4me3 and H3K27me3) [5]. Here we explore the relation between epigenetic regulation in non-malignant cells and neoplastic cells of various origin and demonstrate that non-malignant cells have a low degree of promoter methylation and are strongly marked by H3K27me3 in the SOX11 promoter, independent on investigated cell lineage. Recently, several reports have suggested a crosstalk between DNA methylation and H3K27me3. It has been shown that several genes marked with H3K27me3 undergo de novo methylation in cancer [9]. In the B-cell lineage, Velichutina et al. observed that several EZH2 target genes involved in cellular growth, proliferation and differentiation become methylated in diffuse large B-cell lymphomas [46]. Additionally, Vire et al. demonstrated a physical interaction between 

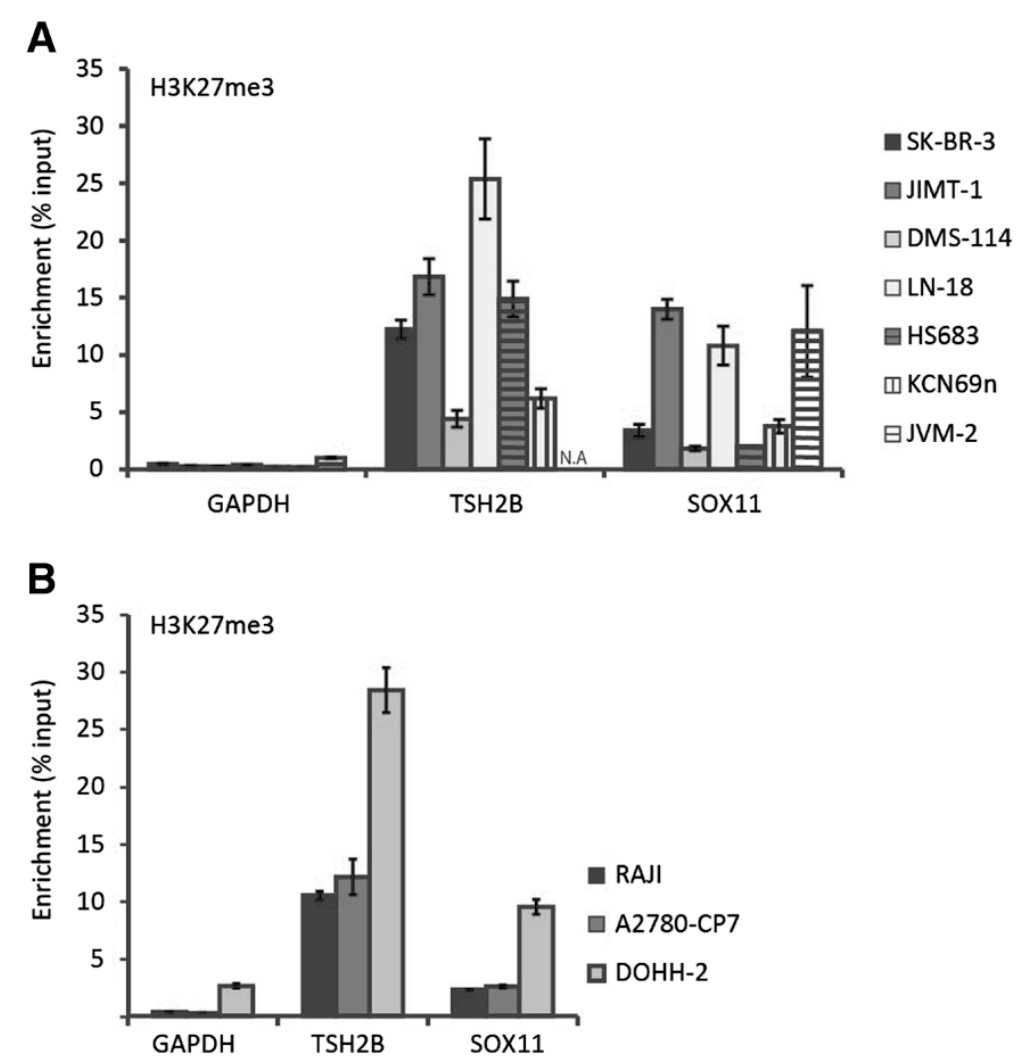

Figure 5 Enrichment of H3K27me3 within the SOX11 promoter. Histone methylation of lysine 27 on histone 3 (H3K27me3) was assessed using chromatin immunoprecipitation and RT-qPCR for GAPDH (negative control), TSH2B (positive control) and SOX11. (A) Enrichment of H3K27me3 in unmethylated cell lines lacking SOX11. (B) Enrichment of H3K27me3 in methylated cell lines lacking SOX11.

DNA methyltransferases and EZH2 [47]. In agreement with this, SOX11 has been reported to be strongly methylated in most B-cell lymphomas [17], in nasopharyngeal carcinomas [19] and in bladder cancer [20]. This prompted us to further investigate the epigenetic regulation of $S O X 11$ in solid tumors.

Our data show that the pattern of SOX11 methylation is more diverse within solid tumor types, compared to within B-cell lymphomas. Within each investigated tumor entity, SOX11 could be unmethylated with or without protein expression or show a varying degree of methylation reflecting a large degree of inter-tumor heterogeneity. Interestingly, SOX11 methylation correlates to ER positivity in breast cancer patients. The difference in epigenetic regulation related to breast cancer hormone status has previously been demonstrated by Müller et al. who showed difference in HDAC expression between ER positive and negative tumors [48]. In contrast to cell lines derived from solid tumors, B cell lymphoma cell lines show similar methylation pattern within each subtype of disease.

DNA microarray studies have shown that HDAC inhibitors induce selective changes in gene expression only affecting a small fraction of genes (2-10\%) [49-51]. As
SOX11 has shown to have a functional role and prognostic relevance in multiple cancer entities, we further investigated the potential to re-express SOX11 using epigenetic drugs. Using the HDAC inhibitors vorinostat and TSA, we show that SOX11 could be re-expressed in three out of five unmethylated cell lines but not in methylated cell lines, suggesting that promoter methylation protects the chromatin from being acetylated and the gene de-methylated and expressed. Furthermore, TSA and vorinostat treatment was shown to decrease the expression of EZH2 in cell lines that re-expressed SOX11, but not in others, further supporting an important role of $\mathrm{EZH} 2$ and $\mathrm{H} 3 \mathrm{~K} 27 \mathrm{me} 3$ methylation in the maintenance of SOX11 silencing. Interestingly, Tiwari et al. recently demonstrated that SOX4, which share 91\% sequence homology to SOX11 within the DNA binding domain [52], regulate the expression of $\mathrm{EZH} 2$ in mouse mammary epithelial and breast cancer cells [53]. However, using the EZH2 inhibitor GSK343, we show that decreased levels of EZH2 are not enough to re-express SOX11. Thus, as recently suggested by Helin et al. [54], H3K27me3 may be a passive mark of un-transcribed genes, and other epigenetic- or transcription factors may initiate the regulation. The re-expression using HDAC 


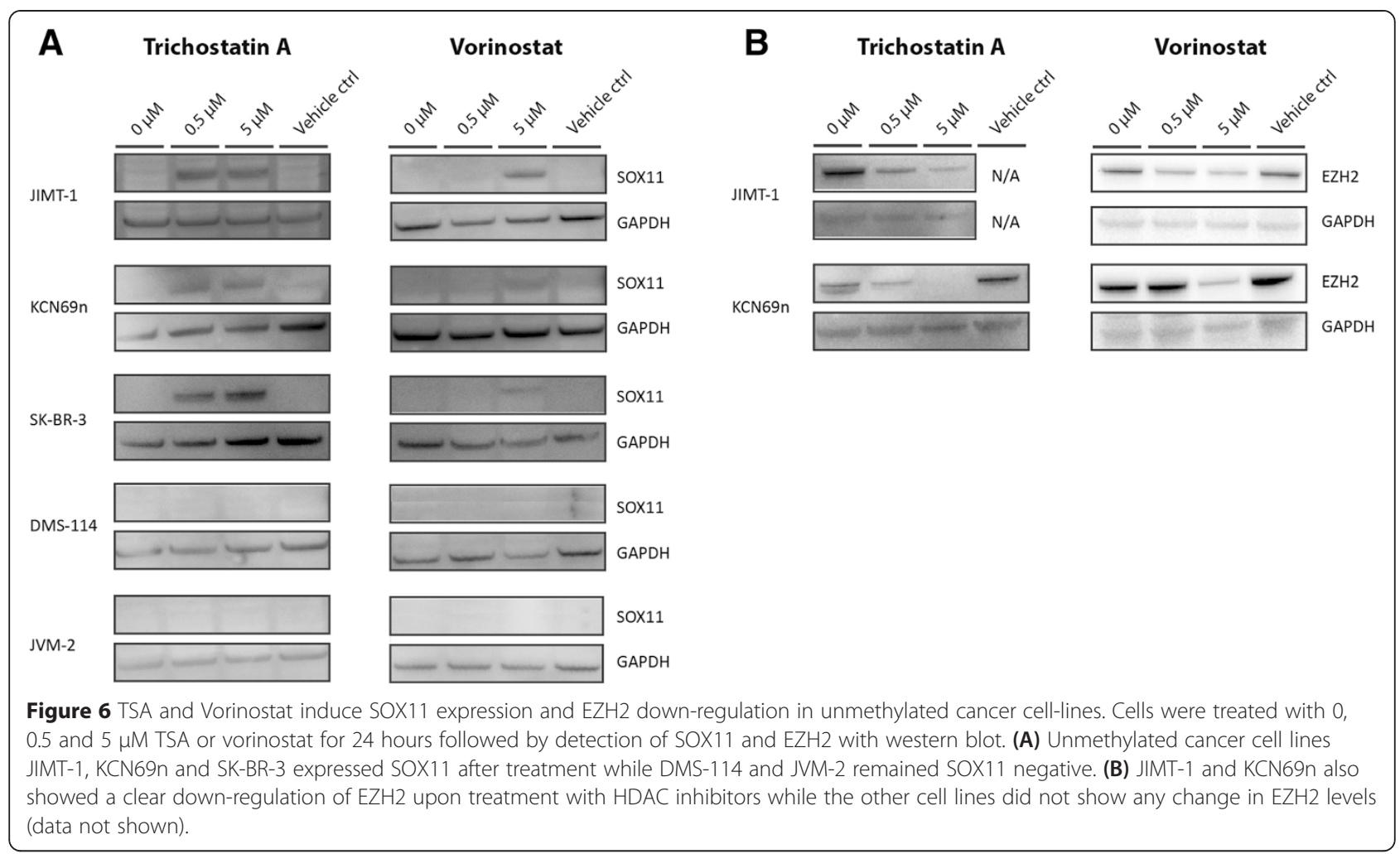

but not EZH2 inhibitors, demonstrate that, in addition to methylation and $\mathrm{H} 3 \mathrm{~K} 27 \mathrm{me} 3$, also acetylation is important in the regulation of SOX11. Vorinostat and TSA inhibit a broad class of HDACs (HDAC1-4, HDAC6-7, and HDAC9) [55] and further investigations are needed to clarify which of these that control SOX11 expression. Although SOX11 was not re-expressed in methylated cell lines, an interaction between HDACs and CpG binding proteins has been demonstrated [56] and HDAC inhibitors have been reported to down regulate the expression of DNA methyl transferases [57].

\section{Conclusions}

To assess the relation between epigenetic regulation of SOX11 in non-malignant tissue, lymphoid and solid malignancies, we investigated methylation and H3K27me3 enrichment at the SOX11 promoter in populations of non-malignant B-cells and fibroblast cells compared to neoplastic cell lines of various origin. In non-malignant cells, SOX11 is strongly marked by enrichment of H3K27me3 while tumors in general show promoter DNA methylation. Of interest, homogeneous methylation of the SOX11 promoter is more frequently observed in lymphomas compared to solid tumors. Analysis of H3K27me3 enrichment in neoplastic cells show that cell lines with an unmethylated SOX11 promoter are strongly marked by H3K27me3, while methylated cell lines are associated with decreased H3K27me3 enrichment, indicating co-regulation of polycomb complex and DNA methyltransferases. We further show that down-regulation of $\mathrm{EZH} 2$ alone do not induce SOX11 expression but that clinically relevant HDAC inhibitors down-regulate EZH2 and induce SOX11 expression. Thus, H3K27me3 in combination with histone acetylation play an important role in SOX11 regulation, and emphasize the need to investigate the potential functional role of SOX11 upon epigenetic treatment and subsequent re-expression in patients with hematological or solid malignancies.

\section{Additional files}

Additional file 1: Detailed description of cell lines, provider and culture media.

Additional file 2: Overview of analyzed CpG sites with MS-MCA, MethyLight and pyrosequencing.

Additional file 3: Interpretation of MS-MCA results.

Additional file 4: Pyrosequencing of $28 \mathrm{CpG}$ sites in low-to-medium methylated cell-lines.

Additional file 5: Western blot showing treatment of SoX11 methylated cell-lines with TSA and SOX11 unmethylated and methylated cell-lines with GSK343.

Competing interests

A patent has previously been filed on the diagnostic, prognostic and therapeutic use of SOX11 in B cell lymphomas. 


\section{Authors' contributions}

LN performed experimental work including gene expression and histone ChIP as well as writing the manuscript. EA performed DNA methylation experiments in relation the malignant cells. VK FACS sorted non-malignant Bcells. EG FACS sorted non-malignant B-cells and performed DNA methylation experiments. $\mathrm{KH}$ and MR were responsible for analysis and interpretation of the breast cancer data. PG was involved in the design of the methylation assays. SE was responsible for the design of the study, interpretation of the data and writing the manuscript. All authors read and approved the final manuscript.

\section{Acknowledgements}

The study was supported by the Lund Institute of Technology (LTH), Cancerfonden, Vetenskapsrådet, Crafoord foundation, BioCARE - a strategic program for Cancer Research at Lund and Gothenburg Universities and CREATE Health.

\section{Author details}

'Department of Immunotechnology, CREATE Health, Lund University, Lund, Sweden. ${ }^{2}$ Danish Cancer Society Research Center, Copenhagen, Denmark. ${ }^{3}$ Department of Oncology and Pathology, Clinical Sciences, Lund University, Skåne University Hospital, Lund, Sweden.

\section{Received: 14 July 2014 Accepted: 18 March 2015}

Published online: 12 April 2015

\section{References}

1. Kiefer JC. Back to basics: Sox genes. Dev Dyn. 2007;236(8):2356-66

2. Lefebvre $V$, Dumitriu B, Penzo-Mendez A, Han Y, Pallavi B. Control of cell fate and differentiation by Sry-related high-mobility-group box (Sox) transcription factors. Int J Biochem Cell Biol. 2007:39(12):2195-214

3. Wang Y, Lin L, Lai H, Parada LF, Lei L. Transcription factor Sox 11 is essential for both embryonic and adult neurogenesis. Dev Dyn. 2013;242(6):638-53.

4. Penzo-Mendez Al. Critical roles for SoxC transcription factors in development and cancer. Int J Biochem Cell Biol. 2010;42(3):425-8.

5. Mikkelsen TS, Ku M, Jaffe DB, Issac B, Lieberman E, Giannoukos G, et al. Genome-wide maps of chromatin state in pluripotent and lineage-committed cells. Nature. 2007:448(7153):553-60.

6. Bernstein BE, Mikkelsen TS, Xie X, Kamal M, Huebert DJ, Cuff J, et al. A bivalent chromatin structure marks key developmental genes in embryonic stem cells. Cell. 2006;125(2):315-26.

7. Richly H, Aloia L, Di Croce L. Roles of the Polycomb group proteins in stem cells and cancer. Cell Death Dis. 2011;2:e204.

8. Vegliante MC, Royo C, Palomero J, Salaverria I, Balint B, Martin-Guerrero I, et al. Epigenetic activation of SOX11 in lymphoid neoplasms by histone modifications. PLoS One. 2011:6(6):e21382.

9. Schlesinger $Y$, Straussman R, Keshet I, Farkash S, Hecht M, Zimmerman J, et al. Polycomb-mediated methylation on Lys 27 of histone $\mathrm{H3}$ pre-marks genes for de novo methylation in cancer. Nat Genet. 2007:39(2):232-6.

10. Keshet I, Schlesinger $Y$, Farkash S, Rand E, Hecht M, Segal E, et al. Evidence for an instructive mechanism of de novo methylation in cancer cells. Nat Genet. 2006;38(2):149-53.

11. Weigle B, Ebner R, Temme A, Schwind S, Schmitz M, Kiessling A, et al. Highly specific overexpression of the transcription factor SOX11 in human malignant gliomas. Oncol Rep. 2005;13(1):139-44.

12. de Bont JM, Kros JM, Passier MMCJ, Reddingius RE, Smitt PAES, Luider TM, et al. Differential expression and prognostic significance of SOX genes in pediatric medulloblastoma and ependymoma identified by microarray analysis. Neuro-Oncology. 2008;10(5):648-60

13. Ek S, Dictor $M$, Jerkeman $M$, Jirstrom K, Borrebaeck CA. Nuclear expression of the non B-cell lineage Sox11 transcription factor identifies mantle cell lymphoma. Blood. 2008;111(2):800-5.

14. Dictor M, Ek S, Sundberg M, Warenholt J, Gyorgy C, Sernbo S, et al. Strong lymphoid nuclear expression of SOX11 transcription factor defines lymphoblastic neoplasms, mantle cell lymphoma and Burkitt's lymphoma. Haematologica. 2009:94(11):1563-8.

15. Brennan DJ, Ek S, Doyle E, Drew T, Foley M, Flannelly G, et al. The transcription factor Sox 11 is a prognostic factor for improved recurrence-free survival in epithelial ovarian cancer. Eur J Cancer. 2009;45(8):1510-7.
16. Zvelebil M, Oliemuller E, Gao Q, Wansbury O, Mackay A, Kendrick H, et al. Embryonic mammary signature subsets are activated in Brca1-/- and basal-like breast cancers. Breast Cancer Res. 2013;15(2):R25

17. Gustavsson E, Sernbo S, Andersson E, Brennan DJ, Dictor M, Jerkeman M et al. SOX11 expression correlates to promoter methylation and regulates tumor growth in hematopoietic malignancies. Mol Cancer. 2010:9:187.

18. Nordström L, Sernbo S, Eden P, Grønbæk K, Kolstad A, Räty R, et al. SOX11 and TP53 add prognostic information to MIPI in a homogenously treated cohort of mantle cell lymphoma - a Nordic Lymphoma Group study. Br J Haematol. 2014;166(1):98-108.

19. Zhang S, Li S, Gao JL. Promoter methylation status of the tumor suppressor gene SOX11 is associated with cell growth and invasion in nasopharyngeal carcinoma. Cancer Cell Int. 2013;13(1):109.

20. Chung W, Bondaruk J, Jelinek J, Lotan Y, Liang S, Czerniak B, et al. Detection of bladder cancer using novel DNA methylation biomarkers in urine sediments. Cancer Epidemiol Biomarkers Prev. 2011;20(7):1483-91.

21. Bock C, Reither S, Mikeska T, Paulsen M, Walter J, Lengauer T. BiQ Analyzer: visualization and quality control for DNA methylation data from bisulfite sequencing. Bioinformatics. 2005;21(21):4067-8

22. Rohde C, Zhang Y, Jurkowski TP, Stamerjohanns $H$, Reinhardt R, Jeltsch A. Bisulfite sequencing Data Presentation and Compilation (BDPC) web servera useful tool for DNA methylation analysis. Nucleic Acids Res. 2008;36(5):e34.

23. Dedeurwaerder S, Defrance M, Calonne E, Denis H, Sotiriou C, Fuks F. Evaluation of the Infinium Methylation 450K technology. Epigenomics. 2011;3(6):771-84.

24. Bernstein BE, Birney E, Dunham I, Green ED, Gunter C, Snyder M. An integrated encyclopedia of DNA elements in the human genome. Nature. 2012;489(7414):57-74.

25. Worm J, Aggerholm A, Guldberg P. In-tube DNA methylation profiling by fluorescence melting curve analysis. Clin Chem. 2001:47(7):1183-9.

26. Trinh BN, Long TI, Laird PW. DNA methylation analysis by MethyLight technology. Methods. 2001;25(4):456-62.

27. Weisenberger DJ, Campan M, Long TI, Kim M, Woods C, Fiala E, et al. Analysis of repetitive element DNA methylation by MethyLight. Nucleic Acids Res. 2005;33(21):6823-36.

28. Nordstrom L, Andreasson U, Jerkeman M, Dictor M, Borrebaeck C, Ek S. Expanded clinical and experimental use of SOX11 - using a monoclonal antibody. BMC Cancer. 2012;12(1):269.

29. Wasik AM, Lord M, Wang X, Zong F, Andersson P, Kimby E, et al. SOXC transcription factors in mantle cell lymphoma: the role of promoter methylation in SOX11 expression. Sci Rep. 2013;3:1400.

30. Ou JN, Torrisani J, Unterberger A, Provencal N, Shikimi K, Karimi M, et al. Histone deacetylase inhibitor Trichostatin A induces global and gene-specific DNA demethylation in human cancer cell lines. Biochem Pharmacol. 2007;73(9):1297-307

31. Arzenani MK, Zade AE, Ming Y, Vijverberg SJ, Zhang Z, Khan Z, et al Genomic DNA hypomethylation by histone deacetylase inhibition implicates DNMT1 nuclear dynamics. Mol Cell Biol. 2011;31(19):4119-28.

32. Wu LP, Wang X, Li L, Zhao Y, Lu SL, Yu Y, et al. Histone deacetylase inhibitor depsipeptide activates silenced genes through decreasing both $\mathrm{CpG}$ and H3K9 methylation on the promoter. Mol Cell Biol. 2008;28(10):3219-35.

33. Dawson MA, Kouzarides T. Cancer epigenetics: from mechanism to therapy. Cell. 2012;150(1):12-27.

34. Issa JP, Kantariian HM, Kirkpatrick P. Azacitidine. Nat Rev Drug Discov. 2005;4(4):275-6.

35. Kantarjian H, Issa JP, Rosenfeld CS, Bennett JM, Albitar M, DiPersio J, et al. Decitabine improves patient outcomes in myelodysplastic syndromes: results of a phase III randomized study. Cancer. 2006;106(8):1794-803.

36. Mann BS, Johnson JR, Cohen MH, Justice R, Pazdur R. FDA approval summary: vorinostat for treatment of advanced primary cutaneous T-cell lymphoma. Oncologist. 2007;12(10):1247-52.

37. Piekarz RL, Frye R, Turner M, Wright JJ, Allen SL, Kirschbaum MH, et al. Phase II multi-institutional trial of the histone deacetylase inhibitor romidepsin as monotherapy for patients with cutaneous T-cell lymphoma. J Clin Oncol. 2009;27(32):5410-7.

38. Culmes M, Eckstein HH, Burgkart R, Nussler AK, Guenther M, Wagner E, et al. Endothelial differentiation of adipose-derived mesenchymal stem cells is improved by epigenetic modifying drug BIX-01294. Eur J Cell Biol. 2012;92(2):70-9.

39. Salvador MA, Wicinski J, Cabaud $O$, Toiron $Y$, Finetti $P$, Josselin $E$, et al. The histone deacetylase inhibitor abexinostat induces cancer stem cells differentiation in breast cancer with low Xist expression. Clin Cancer Res. 2013;19(23):6520-31. 
40. Svechnikova I, Almqvist PM, Ekstrom TJ. HDAC inhibitors effectively induce cell type-specific differentiation in human glioblastoma cell lines of different origin. Int J Oncol. 2008;32(4):821-7

41. Nygren L, Baumgartner Wennerholm S, Klimkowska M, Christensson B, Kimby E, Sander B. Prognostic role of SOX11 in a population-based cohort of mantle cell lymphoma. Blood. 2012;119(18):4215-23.

42. Wang X, Asplund AC, Porwit A, Flygare J, Smith Cl, Christensson B, et al. The subcellular Sox 11 distribution pattern identifies subsets of mantle cell Iymphoma: correlation to overall survival. Br J Haematol. 2008;143(2):248-52.

43. Fernandez V, Salamero O, Espinet B, Sole F, Royo C, Navarro A, et al. Genomic and gene expression profiling defines indolent forms of mantle cell lymphoma. Cancer Res. 2010;70:1408-18. 2010/02/04 edn.

44. Navarro A, Clot G, Royo C, Jares P, Hadzidimitriou A, Agathangelidis A, et al. Molecular subsets of mantle cell lymphoma defined by the IGHV mutational status and SOX11 expression have distinct biologic and clinical features. Cancer Res. 2012;72(20):5307-16.

45. Korkolopoulou P, Levidou G, El-Habr EA, Adamopoulos C, Fragkou P, Boviatsis E, et al. Sox11 expression in astrocytic gliomas: correlation with nestin/c-Met/ IDH1-R132H expression phenotypes, p-Stat-3 and survival. Br J Cancer. 2013;108(10):2142-52.

46. Velichutina I, Shaknovich R, Geng H, Johnson NA, Gascoyne RD, Melnick AM et al. EZH2-mediated epigenetic silencing in germinal center B cells contributes to proliferation and lymphomagenesis. Blood. 2010;116(24):5247-55.

47. Vire E, Brenner C, Deplus R, Blanchon L, Fraga M, Didelot C, et al. The Polycomb group protein EZH2 directly controls DNA methylation. Nature. 2006:439(7078):871-4.

48. Muller BM, Jana L, Kasajima A, Lehmann A, Prinzler J, Budczies J, et al. Differential expression of histone deacetylases HDAC1, 2 and 3 in human breast cancer-overexpression of HDAC2 and HDAC3 is associated with clinicopathological indicators of disease progression. BMC Cancer. 2013;13:215.

49. Van Lint C, Emiliani S, Verdin E. The expression of a small fraction of cellular genes is changed in response to histone hyperacetylation. Gene Expr. 1996;5(4-5):245-53.

50. Glaser KB, Staver MJ, Waring JF, Stender J, Ulrich RG, Davidsen SK. Gene expression profiling of multiple histone deacetylase (HDAC) inhibitors: defining a common gene set produced by HDAC inhibition in T24 and MDA carcinoma cell lines. Mol Cancer Ther. 2003:2(2):151-63.

51. LaBonte MJ, Wilson PM, Fazzone W, Groshen S, Lenz HJ, Ladner RD. DNA microarray profiling of genes differentially regulated by the histone deacetylase inhibitors vorinostat and LBH589 in colon cancer cell lines. BMC Med Genomics. 2009;2:67.

52. Dy P, Penzo-Mendez A, Wang H, Pedraza CE, Macklin WB, Lefebvre V. The three SoxC proteins-Sox4, Sox11 and Sox12-exhibit overlapping expression patterns and molecular properties. Nucleic Acids Res. 2008;36(9):3101-17.

53. Tiwari N, Tiwari VK, Waldmeier L, Balwierz PJ, Arnold P, Pachkov M, et al. Sox4 is a master regulator of epithelial-mesenchymal transition by controlling Ezh2 expression and epigenetic reprogramming. Cancer Cell. 2013;23(6):768-83.

54. Riising EM, Comet I, Leblanc B, Wu X, Johansen JV, Helin K. Gene silencing triggers polycomb repressive complex 2 recruitment to $\mathrm{CpG}$ islands genome wide. Mol Cell. 2014;55(3):347-60.

55. Witt O, Deubzer HE, Milde T, Oehme I. HDAC family: What are the cancer relevant targets? Cancer Lett. 2009;277(1):8-21.

56. Jones PL, Veenstra GJ, Wade PA, Vermaak D, Kass SU, Landsberger N, et al. Methylated DNA and MeCP2 recruit histone deacetylase to repress transcription. Nat Genet. 1998;19(2):187-91.

57. Zhou Q, Agoston AT, Atadja P, Nelson WG, Davidson NE. Inhibition of histone deacetylases promotes ubiquitin-dependent proteasomal degradation of DNA methyltransferase 1 in human breast cancer cells. Mol Cancer Res. 2008;6(5):873-83.

\section{Submit your next manuscript to BioMed Central and take full advantage of:}

- Convenient online submission

- Thorough peer review

- No space constraints or color figure charges

- Immediate publication on acceptance

- Inclusion in PubMed, CAS, Scopus and Google Scholar

- Research which is freely available for redistribution

Submit your manuscript at www.biomedcentral.com/submit 